\title{
VIEWING PARALLEL PROJECTION METHODS AS SEQUENTIAL ONES IN CONVEX FEASIBILITY PROBLEMS
}

\author{
G. CROMBEZ
}

\begin{abstract}
We show that the parallel projection method with variable weights and one variable relaxation coefficient for obtaining a point in the intersection of a finite number of closed convex sets in a given Hilbert space may be interpreted as a semi-alternating sequential projection method in a suitably newly constructed Hilbert space. As such, convergence results for the parallel projection method may be derived from those which may be constructed in the semi-alternating sequential case.
\end{abstract}

\section{INTRODUCTION}

Finding a point in the intersection of a finite number of closed convex sets $\left\{C_{j}\right\}_{j=1}^{r}$ in $\mathbb{R}^{n}$ or, more generally, in a Hilbert space is a typical problem in different branches of applied mathematics. For instance, when solving a large consistent system of linear equations or inequalities in $\mathbb{R}^{n}$, each block of equations or inequalities constrains the solution to lie in a convex set $C_{j}$; in computer tomography with limited data, in which an unknown image has to be reconstructed from a priori knowledge and from measured results, each piece of information gives a constraint which often gives rise to a convex set $C_{j}$ in $L^{2}\left(\mathbb{R}^{2}\right)$ to which the unknown image should belong. For other examples we refer to $[6, \S 4]$.

Since usually it is not possible to obtain a point in $C^{*}=\bigcap_{j=1}^{r} C_{j}$ in a direct manner, an iterative procedure using the projections $P_{j}$ onto the different sets $C_{j}$ is created where, starting from some point $x_{0}$ in the Hilbert space, a sequence $\left\{x_{i}\right\}_{i=1}^{+\infty}$ is constructed that converges to a point in $C^{*}$. In the simplest form of the oldest method, which may be called the sequential method, a new point $x_{k+1}$ in the sequence is obtained from $x_{k}$ by completing a cycle of projections onto the individual sets $C_{j}$, i.e., $x_{k+1}=P_{r} P_{r-1} \cdots P_{2} P_{1} x_{k}$. On the other hand, when for the computations a parallel computer is available, it may be favourable to use another method (which may be called the parallel method) of constructing the sequence $\left\{x_{i}\right\}_{i=1}^{+\infty}$. To this end, at each iteration step $i$ a set of $r$ nonnegative real numbers $\left\{\mu_{i}(j)\right\}_{j=1}^{r}$ with $\sum_{j=1}^{r} \mu_{i}(j)=1$ are chosen (these are called the weights) and, again in the simplest form, the new point $x_{k+1}$

Received by the editors April 5, 1994 and, in revised form, May 4, 1994; originally communicated to the Proceedings of the AMS by Palle E. T. Jorgensen.

1991 Mathematics Subject Classification. Primary 46C10, 47H09, 68U10.

Key words and phrases. Convex set, parallel projection method, sequential projection method, convex feasibility problem, block-iterative projection method. 
in the iteration may be obtained from $x_{k}$ by $x_{k+1}=\sum_{j=1}^{r} \mu_{k+1}(j) P_{j} x_{k}$. Since at first sight both methods are completely different from each other, convergence results of the constructed sequences $\left\{x_{i}\right\}_{i=1}^{+\infty}$ have to be proven for both cases separately. As main results of this paper we show that the parallel method with variable weights (and one variable relaxation coefficient) in a Hilbert space may be viewed as a suitable sequential projection method with possibly an infinite number of convex sets in a convenient new Hilbert space (Theorem 1), and that convergence of the corresponding new sequence in the sequential method entails convergence of the parallel sequence (Theorem 2). This leads to different interesting conclusions, and to interesting possibilities for further research:

(i) It shows that the parallel method with weights which stay fixed at each iteration always converges, since it corresponds to a sequential method in which only two convex sets are involved. On the other hand, for the parallel method with variable weights at each iteration step, convergence will only be guaranteed under some conditions on the weights, since in this case the corresponding sequential method involves an infinite number of convex sets.

(ii) Since the parallel method is in fact a disguised sequential one, those conditions on the weights referred to in (i) may be derived from convergence results in the sequential method with an infinite number of convex sets, which is nowadays a subject of intensive research.

(iii) Possibly the most interesting forthcoming results will handle the manner in which the variable weights in the parallel method have to be chosen in order to optimize the speed of convergence; a first result in this direction, based on this paper, has been submitted for publication [3].

\section{MATHEMATICAL PRELIMINARIES}

Suppose that in the real Hilbert space $H \quad r$ closed convex sets $C_{j} \quad(j=$ $1, \ldots, r)$ are given, whose intersection $C^{*}=\bigcap_{j=1}^{r} C_{j}$ may be empty or nonempty. The parallel projection method (also called block-iterative projection method), with variable weights $\mu_{i}(j)$ where $i$ denotes the iteration index $(i=$ $1,2, \ldots)$ and where $j=1,2, \ldots, r$, and with one variable relaxation coefficient $\lambda_{i} \quad(i=1,2, \ldots)$, is defined as follows in order to obtain a point in $C^{*}$ (when $\left.C^{*} \neq \varnothing\right)$, or a suitable point in $H$ (when $C^{*}=\varnothing$ ), in an iterative manner: starting from an element $x_{0}$ in $H$, we consider the projections $P_{j} x_{0}$ of $x_{0}$ onto the different sets $C_{j}$; choosing $r$ nonnegative real numbers $\mu_{1}(1), \mu_{1}(2), \ldots, \mu_{1}(r)$ (the weights) such that $\sum_{j=1}^{r} \mu_{1}(j)=1$, and one strictly positive real number $\lambda_{1}$, the first iteration point $x_{1}$ is given by

$$
x_{1}=x_{0}+\lambda_{1} \sum_{j=1}^{r} \mu_{1}(j)\left(P_{j} x_{0}-x_{0}\right) ;
$$

in a general way, when the point $x_{k}$ in the iteration has been obtained, the next iteration point $x_{k+1}$ is given by

$$
x_{k+1}=x_{k}+\lambda_{k+1} \sum_{j=1}^{r} \mu_{k+1}(j)\left(P_{j} x_{k}-x_{k}\right) \text {, }
$$


with $\left\{\mu_{k+1}(j)\right\}_{j=1}^{r}$ a set of $r$ nonnegative real numbers such that $\sum_{j=1}^{r} \mu_{k+1}(j)$ $=1$, and with $\lambda_{k+1}$ a strictly positive real number.

In several papers (e.g., $[1,2,5])$ it has been shown that, under suitable conditions about the variable weights and the variable relaxation coefficient, the sequence $\left\{x_{k}\right\}_{k=1}^{\infty}$ is convergent, either weakly or in norm, to a point in the intersection $C^{*}$ (when $C^{*} \neq \varnothing$ ), or to some point whose position is "optimal" with respect to the different sets $C_{j}$.

The mentioned parallel projection method, which originated in the beginning of the eighties, was a logical outgrowth of the sequential projection method which has been known for a much longer time [6]. In the simplest form of the sequential method the pure projections $P_{1}, \ldots, P_{r}$ are combined to form the operator $T=P_{r} P_{r-1}, \ldots, P_{2} P_{1}$; starting from a given element $x_{0}$ in $H$, the iteration sequence $\left\{x_{k}\right\}_{k=1}^{\infty}$ is given by $x_{k+1}=T x_{k}$. More generally, when introducing variable relaxation coefficients $\lambda_{i}(1), \lambda_{i}(2), \ldots, \lambda_{i}(r)$ $(i=1,2, \ldots)$, and denoting by 1 the identity operator on $H$, the operators $T_{i}(j)(j=1, \ldots, r)$ may be defined by

$$
T_{i}(j)=\mathbf{1}+\lambda_{i}(j)\left(P_{j}-\mathbf{1}\right) ;
$$

putting $T_{i}=T_{i}(r) T_{i}(r-1) \cdots T_{i}(2) T_{i}(1)$, the iteration sequence is given by $x_{1}=T_{1} x_{0}, x_{2}=T_{2} x_{1}, \ldots, x_{k+1}=T_{k+1}\left(x_{k}\right), \ldots$, where again $x_{0}$ is a given starting element. Several variants of the sequential method exist; investigation of convergence when an infinite number of closed convex sets $C_{j}$ are involved has been done by Ottavy [7].

In [8], Pierra showed that, when using in the parallel projection method weights which stay fixed during the iteration and with $\lambda_{i}=1$ for all $i=$ $1,2, \ldots$ (i.e., such that $\left.x_{k+1}=\sum_{j=1}^{r} \mu(j) P_{j}\left(x_{k}\right)\right)$, the parallel method may be seen as a sequential method of projections onto two convex sets in a suitable product Hilbert space. Recently, De Pierro [4] tried to generalize the result of Pierra for the case of a parallel projection method with pure projections (i.e., without using a relaxation coefficient) but with variable weights.

In this paper, we refine the ideas appearing in [4] in order to show that the parallel projection method with variable weights and one variable relaxation coefficient may be viewed as a suitable sequential projection method in a convenient Hilbert space. As such, convergence results pertaining to the proper sequential projection method may immediately be transferred to the parallel case.

\section{Construction of the CONVENiENT Hilbert SPACE}

Let the given Hilbert space $H$ be endowed with the inner product $\langle$,$\rangle and$ norm \|\| derived from $\langle$,$\rangle ; the intersection C^{*}=\bigcap_{j=1}^{r} C_{j}$ of the closed convex sets $C_{j}(j=1, \ldots, r)$ may be empty or nonempty.

For $i=1,2, \ldots$ we denote by $\left(N_{r}, \mu_{i}\right)$ the set $\{1,2, \ldots, r\}$ endowed with a (probability) measure $\mu_{i}$ such that $\mu_{i}(j) \geq 0$ for $j=1,2, \ldots, r$ and $\sum_{j=1}^{r} \mu_{i}(j)=1$. These nonnegative numbers $\mu_{i}(j)$ denote the weights at the $i$ th iteration step in (1). Consider the closed interval $[0,1]$ with its Borel measurable sets and with the corresponding Lebesgue measure $m$. When $H$ is made into a measurable space by considering the Borel measurable sets corresponding to the norm topology on $H$, let $\mathscr{L}([0,1], H)$ be the set of all 
measurable functions from $[0,1]$ to $H$, and denote by $L^{2}([0,1], H)$ the set of equivalence classes in $\mathscr{L}([0,1], H)$ of functions $\varphi$ in $\mathscr{L}([0,1], H)$ such that

$$
\|\varphi\|^{2} \equiv \int_{0}^{1}\|\varphi(t)\|^{2} d m(t)<\infty .
$$

Putting, for functions $\varphi$ and $\psi$ in $\mathscr{L}([0,1], H)$,

$$
\langle\langle\varphi, \psi\rangle\rangle=\int_{0}^{1}\langle\varphi(t), \psi(t)\rangle d m(t),
$$

we obtain that $L^{2}([0,1], H)$ is a Hilbert space with norm ||| ||| derived from the inner product $\langle\langle\rangle$,$\rangle . Whenever convenient, L^{2}([0,1], H)$ will be denoted for short by $\mathscr{H}$.

For each fixed $i \in\{1,2, \ldots\}$ the nonnegative numbers $\left\{\mu_{i}(j)\right\}_{j=1}^{r}$ are known. Let $T_{i}$ then be a function from $\left(N_{r}, \mu_{i}\right)$ into the set of subsets of $[0,1]$ defined as follows:

$$
\left.\left.T_{i}(1)=\right] 0, \mu_{i}(1)\right],
$$

and, for $j=2,3, \ldots, r$,

$$
\left.\left.T_{i}(j)=\right] \mu_{i}(1)+\cdots+\mu_{i}(j-1), \mu_{i}(1)+\cdots+\mu_{j}(j)\right] .
$$

Together with the singleton number $\{0\}$, the sets $T_{i}(j)(j=1, \ldots, r)$ form a disjoint covering of $[0,1]$; note that some of the sets $T_{i}(j)$ may be empty, depending on the possible zero value of some $\mu_{i}(j)$; in fact, we use the standard convention that for a real number $a$ the half-open interval $] a, a$ ] is empty.

We now introduce in the newly defined Hilbert space $\mathscr{l}$ the subsets $F_{\mu_{i}}$ which, together with one other set, will play the role of the closed convex sets for the sequential projection method which is to be equivalent to the parallel projection method of (1). To this end, we remark that whenever we choose (for a fixed $i \in\{1,2, \ldots\}$, and hence also for a fixed $\mu_{i}$ ) an element $v_{j}$ in each set $C_{j}$ in $H(j=1, \ldots, r)$, a measurable function $\varphi^{i}:[0,1] \rightarrow H$ may be introduced as follows: for $t \in T_{i}(j)$, we put $\varphi^{i}(t)=v_{j}\left(\in C_{j}\right)$; we let $\varphi^{i}(0)$ be that chosen element $v_{k}$ in $C_{k}$ with the smallest index $k \in\{1,2, \ldots, r\}$ such that $T_{i}(k) \neq \varnothing$. As such, the function $\varphi^{i}$ may be seen to be a piecewise constant function defined on $[0,1]$, whose values are lying in some or all of the sets $C_{j}$. Such function $\varphi^{i}$ is clearly square integrable, since

$$
\int_{0}^{1}\left\|\varphi^{i}(t)\right\|^{2} d m(t)=\sum_{j=1}^{r}\left\|v_{j}\right\|^{2} m\left(T_{i}(j)\right)=\sum_{j=1}^{r}\left\|v_{j}\right\|^{2} \mu_{i}(j)<\infty .
$$

Considering the set of all such functions for a fixed $i$ and for all possible choices of elements $v_{j}$ in the different sets $C_{j}$, we denote the set of equivalence classes of these functions in $\mathscr{H}$ by $F_{\mu_{i}}$. For each $i, F_{\mu_{i}}$ is a subset of $\mathscr{H}$.

We finally introduce in $\mathscr{H}$ the "diagonal" $D$, i.e., the set of equivalence classes which correspond to constant functions in $\mathscr{L}([0,1], H)$. Clearly, $D$ is isometric to $H$ by means of the natural imbedding $q: H \rightarrow L^{2}([0,1], H)$ which makes each element $v$ in $H$ correspond to the function $q(v)$ in $L^{2}([0,1], H)$ given by $q(v)(t)=v, \forall t \in[0,1]$. 


\section{RESUlTS AND PROOFS}

Lemma 1. The isometric image $D \equiv q(H)$ of $H$ in $\mathscr{H}$ is a closed linear subspace of $\mathscr{H}$.

Proof. That $D$ is a linear subspace may immediately be verified. To show that $D$ is closed, let $\left\{\varphi_{n}\right\}_{n=1}^{\infty}$ be a sequence in $D$ such that $\varphi_{n} \rightarrow \varphi$ in $\mathscr{H}$. We have to show that there exists a vector $v$ in $H$ such that $\varphi(t)=v, \forall t \in[0,1]$. There exists a sequence of vectors $\left\{v_{n}\right\}_{n=1}^{\infty}$ in $H$ such that, for each fixed $n$, $\varphi_{n}(t)=v_{n}$ for all $t \in[0,1]$. Since the sequence $\left\{\varphi_{n}\right\}_{n=1}^{\infty}$ is a Cauchy sequence in $\mathscr{H}$ we have, for positive integers $m$ and $n$,

$$
\left\|\varphi_{n}-\varphi_{m}\right\|\left\|^{2}=\int_{0}^{1}\right\| v_{n}-v_{m}\left\|^{2} d t=\right\| v_{n}-v_{m} \|^{2},
$$

which tends to zero as $n, m \rightarrow+\infty$. Let $v$ be the limit of the Cauchy sequence $\left\{v_{n}\right\}_{n=1}^{\infty}$ in $H$, and put $\psi(t)=v, \forall t \in[0,1]$. We show that the functions $\varphi$ and $\psi$ are equivalent in $\mathscr{H}$, thereby proving the result. This follows easily, since for all $t \in[0,1]$ we have

$$
\|\varphi(t)-\psi(t)\|^{2} \leq 2\left\|\varphi(t)-v_{n}\right\|^{2}+2\left\|v_{n}-v\right\|^{2},
$$

and hence

$$
\begin{aligned}
\|\varphi-\psi\| \|^{2} & \leq 2 \int_{0}^{1}\left\|\varphi(t)-v_{n}\right\|^{2} d m(t)+2 \int_{0}^{1}\left\|v_{n}-v\right\|^{2} d m(t) \\
& =2\left\|\varphi-\varphi_{n}\right\|\left\|^{2}+2\right\| v_{n}-v \|^{2},
\end{aligned}
$$

which tends to zero for $n \rightarrow+\infty$. Hence $\varphi$ and $\psi$ belong to the same equivalence class.

Lemma 2. Each set $F_{\mu_{i}}$ is a closed convex subset of $\mathscr{H}$.

Proof. Since the functions in $F_{\mu_{i}}$ are piecewise constant on $[0,1]$, taking their values in (some of) the convex sets $C_{1}, \ldots, C_{r}$ in $H$, it is easily verified that $F_{\mu_{i}}$ is convex.

The proof that $F_{\mu_{i}}$ is closed may be compared to the one showing the closedness of $D$, but it is somewhat more involved. Let $\left\{\varphi_{n}\right\}_{n=1}^{\infty}$ be a sequence in $F_{\mu_{i}}$, and suppose that $\varphi_{n} \rightarrow \varphi$ in $\mathscr{H}$. Depending on the values of $\mu_{i}(1), \ldots, \mu_{i}(r)$, each function $\varphi_{n}$ may take on values $v_{n}^{1}, v_{n}^{2}, \ldots, v_{n}^{r}$ in $C_{1}, C_{2}, \ldots, C_{r}$, respectively. For positive integers $n$ and $m$ we have

$$
\begin{aligned}
\left\|\varphi_{n}-\varphi_{m}\right\| \|^{2} & =\sum_{j=1}^{r} \int_{T_{i}(j)}\left\|\varphi_{n}(t)-\varphi_{m}(t)\right\|^{2} d m(t) \\
& =\sum_{j=1}^{r}\left\|v_{n}^{j}-v_{m}^{j}\right\|^{2} \mu_{i}(j) .
\end{aligned}
$$

Since $\left\{\varphi_{n}\right\}_{n=1}^{\infty}$ is a Cauchy sequence in $\mathscr{H}$, the last sum tends to zero for $n, m \rightarrow+\infty$; hence each (possible) sequence $\left\{v_{n}^{j}\right\}_{n=1}^{\infty}$ in $C_{j}$ converges to some vector $v^{j}$ in the closed convex set $C_{j}$. Defining then the function $\psi$ on the subintervals of $[0,1]$ determined by the numbers $\mu_{i}(1), \ldots, \mu_{i}(r)$ by 
$\psi(t)=v^{j}$ for $t \in T_{i}(j)$ (as previously explained), we obtain a function $\psi$ which clearly belongs to $F_{\mu_{i}}$, and which is equivalent to $\varphi$, since

$$
\|\varphi-\psi\|\left\|^{2} \leq 2 \int_{0}^{1}\right\| \varphi(t)-\varphi_{n}(t)\left\|^{2} d m(t)+2 \int_{0}^{1}\right\| \varphi_{n}(t)-\psi(t) \|^{2} d m(t),
$$

and both terms on the right-hand side tend to zero when $n$ goes to infinity.

Now let $v$ be a given element in $H$. For $j=1,2, \ldots, r$ denote by $P_{j} v$ the projection of $v$ onto $C_{j}$. For some fixed iteration index $i$ the subsets $\left\{T_{i}(j)\right\}_{j=1}^{r}$ of $[0,1]$ are defined by the numbers $\left\{\mu_{i}(j)\right\}_{j=1}^{r}$. Define the function $\varphi_{v}:[0,1] \rightarrow H$ as follows: whenever $T_{i}(j) \neq \varnothing$, put $\varphi_{v}(t)=P_{j} v$ for $t \in T_{i}(j) ;$ put $\varphi_{v}(0)=P_{k} v, k$ being the smallest index in $\{1,2, \ldots, r\}$ such that $T_{i}(k) \neq \varnothing$. Clearly, the function $\varphi_{v}$ so defined belongs to $F_{\mu_{i}}$. On the other hand, the imbedding $q(v)$ of $v$ is an element of $\mathscr{H}$, and so we can consider the projection of $q(v)$ onto the closed convex subset $F_{\mu_{i}}$. Denote the projection operator of $\mathscr{H}$ onto $F_{\mu_{i}}$ by $P_{F_{\mu_{i}}}$.

Proposition 1. For a given element $v$ in $H$ with imbedding $q(v)$ in $\mathscr{H}$, the projection of $q(v)$ onto the convex set $F_{\mu_{i}}$ is the function having the values $\left\{P_{j} v\right\}_{j=1}^{r}$ on the subintervals $\left\{T_{i}(j)\right\}_{j=1}^{r}$ of $[0,1]$ defined by the weights $\left\{\mu_{i}(j)\right\}_{j=1}^{r}$; i.e., with the notation from above,

$$
P_{F_{i}}[q(v)]=\varphi_{v} .
$$

Proof. $P_{F_{\mu_{i}}}[q(v)]$ is a function which is piecewise constant on $[0,1]$. Denoting this function for short by $\psi$ we have

$$
\begin{aligned}
\|q(v)-\psi\| \|^{2} & =\min _{\zeta \in F_{\mu_{i}}}\|q(v)-\zeta\|^{2} \\
& =\min _{\zeta \in F_{\mu_{i}}} \sum_{j=1}^{r} \int_{T_{i}(j)}\|v-\zeta(t)\|^{2} d t .
\end{aligned}
$$

Each function $\zeta \in F_{\mu_{i}}$ is constant on a corresponding subinterval $T_{i}(j)$; denoting this constant value by $\zeta_{j}$ we have

$$
\begin{aligned}
\|q(v)-\psi\| \|^{2} & =\min _{\zeta \in F_{\mu_{i}}} \sum_{j=1}^{r}\left\|v-\zeta_{j}\right\|^{2} m\left(T_{i}(j)\right) \\
& =\sum_{j=1}^{r} \min _{\zeta_{j} \in C_{j}}\left\|v-\zeta_{j}\right\|^{2} m\left(T_{i}(j)\right) .
\end{aligned}
$$

Since in each term on the right-hand side the minimum is obtained when $\zeta_{j}=$ $P_{j} v$, we obtain

$$
\begin{aligned}
\|q(v)-\psi\| \|^{2} & =\sum_{j=1}^{r}\left\|v-P_{j} v\right\|^{2} m\left(T_{i}(j)\right) \\
& =\sum_{j=1}^{r}\left\|v-\varphi_{v}(t)\right\|^{2} m\left(T_{i}(j)\right)=\left\|q(v)-\varphi_{v}\right\|^{2} .
\end{aligned}
$$

Hence, the result follows. 
Suppose now that a function $\varphi \in F_{\mu_{i}}$ is given. On the suitable subintervals $T_{i}(j)$ of $[0,1]$ this function $\varphi$ takes on (some of) the values $v_{1}, \ldots, v_{r}$ belonging respectively to the sets $C_{1}, \ldots, C_{r}$ in $H$. We denote by $P_{D}$ the projection operator of $\mathscr{H}$ onto $D$.

Proposition 2. For a given function $\varphi \in F_{\mu_{i}}$ having values $\left\{v_{j}\right\}_{j=1}^{r}$ on the subintervals $\left\{T_{i}(j)\right\}_{j=1}^{r}$ of $[0,1]$ defined by the weights $\left\{\mu_{i}(j)\right\}_{j=1}^{r}$, the projection of $\varphi$ onto $D$ is the imbedding under $q$ of the element $\sum_{j=1}^{r} \mu_{i}(j) v_{j}$ in $H$; i.e.,

$$
P_{D}(\varphi)=q\left(\sum_{j=1}^{r} \mu_{i}(j) v_{j}\right) \text {. }
$$

Proof. We first remember that, for a given $v$ in $H, q(v)$ is the constant function in $D$ such that $q(v)(t)=v$ for all $t \in[0,1]$. Then

$$
\begin{aligned}
\left\|\varphi-P_{D}(\varphi)\right\|^{2} & =\min _{q(v) \in D}\|\varphi-q(v)\| \|^{2} \\
& =\min _{q(v) \in D} \sum_{j=1}^{r} \int_{T_{i}(j)}\left\|v_{j}-v\right\|^{2} d t \\
& =\min _{q(v) \in D} \sum_{j=1}^{r}\left\|v_{j}-v\right\|^{2} m\left(T_{i}(j)\right) .
\end{aligned}
$$

Denoting $\sum_{j=1}^{r}\left\|v_{j}-v\right\|^{2} m\left(T_{i}(j)\right)$ as $f(v)$, we have that its gradient $\operatorname{grad} f(v)$ is given by

$$
\operatorname{grad} f(v)=\sum_{j=1}^{r} 2\left(v_{j}-v\right) m\left(T_{i}(j)\right) .
$$

Since $m\left(T_{i}(j)\right)=\mu_{i}(j)$, we get that $\operatorname{grad} f(v)$ is zero when $v=\sum_{j=1}^{r} \mu_{i}(j) v_{j}$. Hence the foregoing minimum is obtained for the point $q(v)$ in $D$ with $v=$ $\sum_{j=1}^{r} \mu_{i}(j) v_{j}$.

The foregoing propositions give us now the possibility to interpret the parallel projection method in $H$ given by (1) as a sequential projection method in $\mathscr{H}$. To this end, we remark that for a given starting point $x_{0}$ in $H$ for the parallel projection method, the point $q\left(x_{0}\right)$ belongs to $D$. We consider this point $q\left(x_{0}\right)$ as the starting point for a sequential projection method in $\mathscr{H}$, defined in the following manner: put

$$
\widehat{y_{1}}=q\left(x_{0}\right)+\lambda_{1}\left(P_{F_{\mu_{1}}}\left[q\left(x_{0}\right)\right]-q\left(x_{0}\right)\right), \quad \widehat{x_{1}}=P_{D}\left(\widehat{y_{1}}\right) .
$$

In a general manner, when $\widehat{x_{k-1}} \in D$ has already been obtained, put

$$
\widehat{y_{k}}=\widehat{x_{k-1}}+\lambda_{k}\left(P_{F_{\mu_{k}}}\left[\widehat{x_{k-1}}\right]-\widehat{x_{k-1}}\right), \quad \widehat{x_{k}}=P_{D}\left(\widehat{y_{k}}\right) \text {. }
$$

By this semi-alternating sequential projection method with one variable relaxation coefficient we obtain a sequence $q\left(x_{0}\right), \widehat{x_{1}}, \ldots, \widehat{x_{k}}, \ldots$ in $D$. Since all elements in $D$ are in fact derived from elements of $H$, each point $\widehat{x_{k}}$ in the sequence is the image under $q$ of some element $x_{k}$ in $H$.

Theorem 1. If $x_{0}, x_{1}, \ldots, x_{k}, \ldots$ denotes the sequence in $H$ obtained by the parallel method in (1), and $q\left(x_{0}\right), \widehat{x_{1}}, \ldots, \widehat{x_{k}}, \ldots$ is the sequence in $\mathscr{H}$ obtained by the semi-alternating sequential projection method (3), then $\left\{\widehat{x_{k}}\right\}_{k=1}^{\infty}$ is 
exactly obtained by the imbedding in $\mathscr{H}$ of the parallel sequence $\left\{x_{k}\right\}_{k=1}^{\infty}$ in (1); i.e. $\widehat{x_{k}}=q\left(x_{k}\right)$.

Proof. We just consider the first step, since the calculation for the other steps is similar. By Proposition 1 we have that $P_{F_{\mu_{1}}}\left[q\left(x_{0}\right)\right]$ is the function that takes on the values $P_{1} x_{0}, \ldots, P_{r} x_{0}$ on the corresponding subsets $T_{1}(j)$. In view of this result and of the fact that the projection operator $P_{D}$ onto $D$ is linear we obtain, by use of Proposition 2,

$$
\begin{aligned}
\widehat{x_{1}} & =P_{D}\left(\widehat{y_{1}}\right)=q\left(x_{0}\right)+\lambda_{1} P_{D}\left(P_{F_{\mu_{1}}}\left[q\left(x_{0}\right)\right]\right)-\lambda_{1} q\left(x_{0}\right) \\
& =q\left(x_{0}\right)+\lambda_{1} q\left(\sum_{j=1}^{r} \mu_{1}(j) P_{j} x_{0}\right)-\lambda_{1} q\left(x_{0}\right) \\
& =q\left[x_{0}+\lambda_{1} \sum_{j=1}^{r} \mu_{1}(j)\left(P_{j} x_{0}-x_{0}\right)\right]=q\left(x_{1}\right) .
\end{aligned}
$$

An operator notation for the semi-alternating projection method in $\mathscr{H}$ may thus be given as follows: at each iteration step $i$ a variable positive relaxation coefficient $\lambda_{i}$ and $r$ variable nonnegative weights $\left\{\mu_{i}(j)\right\}_{j=1}^{r}$ are given; denoting by id the identity operator on $\mathscr{H}$, the sequence $q\left(x_{0}\right), \widehat{x_{1}}, \ldots, \widehat{x_{k}}, \ldots$ is obtained by repeated application of the (variable) operators $\Pi_{\mu_{i}}^{\lambda_{i}}$ given by

$$
\Pi_{\mu_{i}}^{\lambda_{i}} \equiv P_{D} \circ\left[\mathrm{id}+\lambda_{i}\left(P_{F_{\mu_{i}}}-\mathrm{id}\right)\right]
$$

In particular, when no relaxation coefficients are involved (i.e., $\lambda_{i}=1$ for all $i)$, and when the weights are kept fixed during the iteration, we obtain the result of Pierra.

The importance of viewing the parallel projection method with variable weights as a special sequential one as described above follows readily when we compare the convergence behaviour of the sequence $\left\{x_{k}\right\}_{k=0}^{+\infty}$ as derived from the sequence $\left\{\widehat{x_{k}}\right\}_{k=0}^{+\infty}$. We have

Theorem 2. When the sequence $\left\{\widehat{x_{k}}\right\}_{k=0}^{+\infty}$ in $\mathscr{H}$ converges in norm [weakly], then the sequence $\left\{x_{k}\right\}_{k=0}^{+\infty}$ in $H$ also converges in norm [weakly].

Proof. Suppose first that the sequence $\left\{\widehat{x_{k}}\right\}_{k=0}^{+\infty}$ in $\mathscr{H}$ converges in norm to some $\varphi \in \mathscr{H}$. Since each $\widehat{x_{k}} \in D$ and $D$ is closed in norm, also $\varphi \in D$; so we may put $\varphi=q(w)$ for some $w \in H$. We then have

$$
\begin{aligned}
\left.\left\|\varphi-\widehat{x_{k}}\right\|\right|^{2} & =\left\|q(w)-q\left(x_{k}\right)\right\| \|^{2} \\
& =\int_{0}^{1}\left\|q(w)(t)-q\left(x_{k}\right)(t)\right\|^{2} d m(t) \\
& =\int_{0}^{1}\left\|w-x_{k}\right\|^{2} d m(t)=\left\|w-x_{k}\right\|^{2} .
\end{aligned}
$$

Hence, the sequence $\left\{x_{k}\right\}_{k=0}^{+\infty}$ in $H$ is norm convergent (to $w$ ).

Let us suppose now that the sequence $\left\{\widehat{x_{k}}\right\}_{k=0}^{+\infty}$ in $\mathscr{H}$ is weakly convergent to some $\psi$ in $\mathscr{H}$. Since $D$ is a closed subspace, it is also weakly closed, and so $\psi \in D$. Putting $\psi=q(z)$ for some $z$ in $H$ we have, for all $\varphi \in \mathscr{H}$,

$$
\left\langle\left\langle\widehat{x_{k}}, \varphi\right\rangle\right\rangle \rightarrow\langle\langle q(z), \varphi\rangle\rangle,
$$


or, explicitly,

$$
\int_{0}^{1}\left\langle\widehat{x_{k}}(t), \varphi(t)\right\rangle d m(t) \rightarrow \int_{0}^{1}\langle q(z)(t), \varphi(t)\rangle d m(t) .
$$

Taking for $\varphi$ all elements of the form $q(x)$ for all $x$ in $H$, this leads to

$$
\int_{0}^{1}\left\langle x_{k}, x\right\rangle d m(t) \rightarrow \int_{0}^{1}\langle z, x\rangle d m(t),
$$

i.e., $\left\langle x_{k}, x\right\rangle \rightarrow\langle z, x\rangle$ for all $x \in H$. Hence, the sequence $\left\{x_{k}\right\}_{k=0}^{+\infty}$ is weakly convergent in $H$ (to $z$ ).

We conclude with two properties concerning the intersection of the involved sets.

When $C^{*}=\bigcap_{j=1}^{r} C_{j}$ is nonempty, then also $\left(\bigcap_{i=1}^{\infty} F_{\mu_{i}}\right) \cap D$ is nonempty. Indeed, when $z \in C^{*}$, then $q(z)$ belongs to $D$ and to each $F_{\mu_{i}}$.

When $\left(\bigcap_{i=1}^{\infty} F_{\mu_{i}}\right) \cap D$ is nonempty, then there exists some $q(z)$ in $D$ belonging to each $F_{\mu_{i}}$, where $q(z)(t)=z \in H$ for all $t \in[0,1]$. Assuming that there exist $r$ indexes $g, h, \ldots, s$ such that $\mu_{g}(1) \neq 0, \mu_{h}(2) \neq$ $0, \ldots, \mu_{s}(r) \neq 0$ we have, since $q(z) \in F_{\mu_{g}}, q(z) \in F_{\mu_{h}}, \ldots, q(z) \in F_{\mu_{s}}$, that $\left.\left.q(z)(t) \in C_{1}, \forall t \in\left[0, \mu_{g}(1)\right], q(z)(t) \in C_{2}, \forall t \in\right] \mu_{h}(1), \mu_{h}(1)+\mu_{h}(2)\right], \ldots$. Hence $z \in C_{1}, z \in C_{2}, \ldots, z \in C_{r}$, and so $z \in C^{*}=\bigcap_{j=1}^{r} C_{j}$. So, under the stated assumption also $C^{*}$ is nonempty.

\section{REFERENCES}

1. D. Butnariu and Y. Censor, On the behavior of a block-iterative projection method for solving convex feasibility problems, Internat. J. Computer Math. 43 (1990), 79-94.

2. G. Crombez, Weak and norm convergence of a parallel projection method in Hilbert spaces, Appl. Math. Comput. 56 (1993), 35-48.

3. __ A parallel projection method based on sequential most remote set in convex feasibility problems (submitted for publication).

4. A. R. De Pierro, An extended decomposition through formalization in product spaces, preprint.

5. A. R. De Pierro and A. N. Iussem, A parallel projection method of finding a common point of a family of convex sets, Pesquisa Operacional 5 (1985), 1-20.

6. L. G. Gubin, B. T. Polyak, and E. V. Raik, The method of projections for finding the common point of convex sets, USSR Comput. Math. and Math. Phys. 7 (1967), 1-24.

7. N. Ottavy, Strong convergence of projection-like methods in Hilbert spaces, J. Optim. Theory Appl. 56 (1988), 433-461.

8. G. Pierra, Decomposition through formalization in a product space, Math. Programming 28 (1984), 96-115.

Department of Applied Mathematics and Informatics, University of Ghent, B-9000 GeNT, Belgium

E-mail address: gilbert.crombez@rug.ac.be 